\title{
30-day Mortality in Acute Non-surgical Patients Admitted with Hyponatremia
}

Authors:

L Holland-Bill

SP Ulrichsen ${ }^{1}$

CF Christiansen

T Ring ${ }^{2}$

HT Sørensen ${ }^{1}$

JOL Jørgensen ${ }^{3}$

\section{Department:}

1 Department of Clinical Epidemiology,

Aarhus University Hospital,

Aarhus N, Denmark

2 Department of Nephrology,

Aalborg University Hospital,

Aalborg, Denmark

3 Department of Endocrinology and

Diabetes, Aarhus University Hospital,

Aarhus C, Denmark

\section{Conflicts of interest:}

JOL Jørgensen has received an unrestricted research grant and lecture fees from Otsuka Pharma Scandinavia AB.

The department of Clinical Epidemiology, Aarhus university Hospital receives

funding for other studies from companies in the form of research grants to (and administered by) Aarhus University. None of these studies have any relation to the present study.

Corresponding author's email: louise.bill@dce.au.dk

\section{References:}

1 Incidence and prevalence of hyponatremia. Am J Med. 2006 2 Hyponatremia in hospitalized cancer patients and its impact on clinical outcomes. Am J Kidney Dis. 2012

3 Burden of sodium abnormalities in patients hospitalized for heart failure. Congest Heart Fail. 2011

4 Hyponatremia, Hypernatremia and Mortality in Patients with Chronic Kidney Disease with and without Congestive Heart Failure. Circulation. 2012

5 Serum sodium predicts prognosis in critically ill cirrhotic patients. J Clin Gastroenterol. 2010

\section{Background}

Hyponatremia (serum sodium $<135 \mathrm{mmol} / \mathrm{l}$ ), the most common electrolyte disorder encountered, ${ }^{1}$ has been associated with increased mortality in patients with particularly cancer, heart failure, chronic kidney and liver disease. ${ }^{2-5}$ However, evidence of the prognostic impact in broader populations is scarce, and uncertain due to confounding from preexisting disease.

\section{Objective}

To examine the association between admission-hyponatremia and 30-day mortality in a large heterogeneous population of acute nonsurgical patients.

\section{Methods}

We conducted a population-based cohort study in North and Central Denmark Regions, comprising approximately 1.8 million inhabitants.

- We identified all patients acutely admitted to non-surgical departments from January 1, 2000 to December 31, 2009, for whom serum sodium was measured on the day of admission using individual level linkage of the Danish National Patients Registry, the Danish Civil Registration System and the Clinical Laboratory Information System.

- Admission-hyponatremia was categorized as mild $(130-134.9 \mathrm{mmol} / \mathrm{l})$, moderate $(125-129.9 \mathrm{mmol} / \mathrm{l})$ and severe $(<125 \mathrm{mmol} / \mathrm{l})$

- Thirty-day mortality for normonatremia and levels of admissionhyponatremia were estimated using the Kaplan-Meier method. Hazard ratios (HR) were estimated using a Cox regression model, adjusting for age, gender and Charlson comorbidity level.

Table 1. Characteristics of non-surgical patients stratified on serum sodium level at admission

\begin{tabular}{lccc} 
& $\begin{array}{c}\text { Hyponatremia } \\
(<135 \text { mmol/l) } \\
\mathbf{n}(\%)\end{array}$ & $\begin{array}{c}\text { Normonatremia } \\
(135-145 \mathrm{mmol} / \mathrm{l}) \\
\mathbf{n}(\%)\end{array}$ & $\begin{array}{c}\text { Hypernatremia } \\
(>145 \mathrm{mmol} / \mathrm{l}) \\
\mathbf{n}(\%)\end{array}$ \\
\hline Total & $55,185(100)$ & $241,150(100)$ & $5,976(100)$ \\
\hline Age at hospitalization & & & \\
\hline$<18$ & $1,095(1.9)$ & $6,417(2.7)$ & $201(3.4)$ \\
\hline $18-39$ & $4,209(7.6)$ & $40,447(16.8)$ & $1,147(19.2)$ \\
\hline $40-49$ & $4,327(7.8)$ & $28,575(11.9)$ & $660(11.1)$ \\
\hline $50-59$ & $7,883(14.3)$ & $38,240(15.8)$ & $802(13.4)$ \\
\hline $60-69$ & $11,154(20.2)$ & $42,760(17.7)$ & $794(13.3)$ \\
\hline $70-79$ & $13,243(24.0)$ & $44,599(18.5)$ & $993(16.6)$ \\
\hline $80+$ & $13,274(24.1)$ & $40,148(16.7)$ & $1,379(23.1)$ \\
\hline Gender & & & \\
\hline Female & $29,797(54.0)$ & $119,551(49.6)$ & $2,812(47.1)$ \\
\hline Male & $25,388(46.0)$ & $121,599(50.4)$ & $3,164(52.9)$ \\
\hline Charlson comorbidity $5 c 0 r e$ at hospitalization & \\
\hline 0 & $29,653(53.7)$ & $159,717(66.2)$ & $3,950(66.1)$ \\
\hline $1-2$ & $17,996(32.6)$ & $62,779(26.0)$ & $1,567(26.2)$ \\
\hline $3+$ & $7,536(13.7)$ & $18,654(7.7)$ & $459(7.7)$ \\
\hline
\end{tabular}

Figure

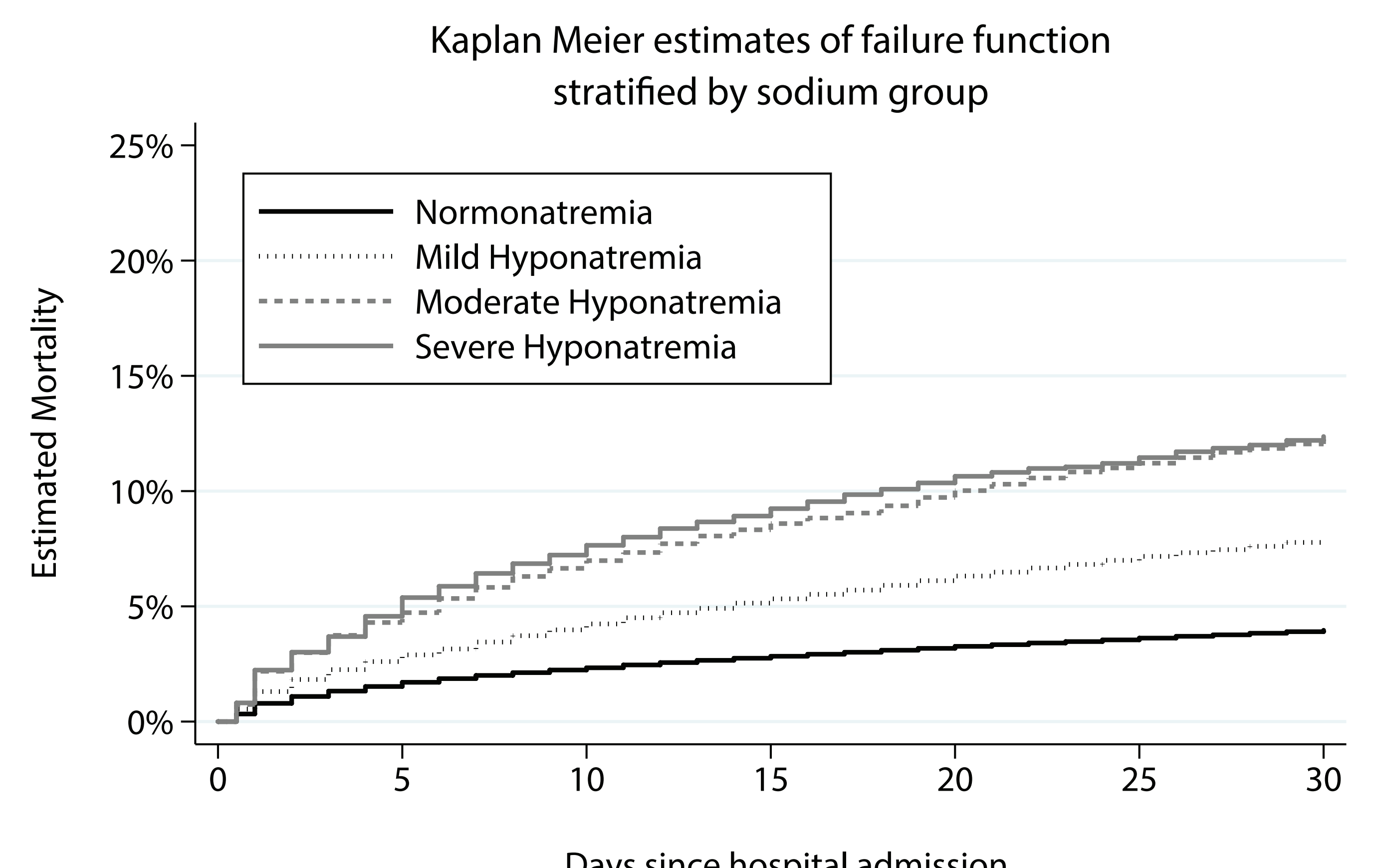

Table 2. 30 day mortality in acute non-surgical patients stratified on serum sodium level at admission.

\begin{tabular}{|c|c|c|c|}
\hline & $\begin{array}{l}\text { Cumulative } \\
\%(95 \% \mathrm{Cl})\end{array}$ & $\begin{array}{l}\text { HR - Crude } \\
(95 \% \mathrm{Cl})\end{array}$ & $\begin{array}{c}\text { HR - } \text { Adjusted }^{\S} \\
(95 \% \mathrm{Cl})\end{array}$ \\
\hline Normonatremia & $4.1(4.0-4.2)$ & Reference & Reference \\
\hline Hyponatremia & $9.7(9.5-10.0)$ & $2.43(2.35-2.52)$ & $1.87(1.80-1.93)$ \\
\hline Mild & $8.6(8.3-8.9)$ & $2.14(2.06-2.23)$ & $1.67(1.61-1.74)$ \\
\hline Moderate & $12.2(11.6-12.9)$ & $3.10(2.93-3.29)$ & $2.27(2.14-2.41)$ \\
\hline Severe & $12.4(11.6-13.2)$ & $3.15(2.92-3.40)$ & $2.35(2.18-2.53)$ \\
\hline
\end{tabular}

Adjusted for age, gender and comorbidity level

\section{Results}

We identified 302,311 acute non-surgical patients, with a sodium measurement on the first day of admission.

- Admission-hyponatremia was present in 55,185 patients (prevalence $=18.3 \%$ )

- Patients with hyponatremia at admission were older (median age 69.1 years (interquartile range: 55.9-79.6) vs. 61.6 years (interquartile range: $44.9-75.5)$ ) and had higher comorbidity levels compared to patients with normonatremia.

- Cumulative 30-day mortality for patients with admissionhyponatremia was 9.7\% (95\% Cl: 9.5-10.0) compared with 4.1\% (95\% Cl: 4.0-4.2) in patients with normonatremia. Mortality was increased throughout the period.

- The adjusted HR was 1.87 (95\% Cl: 1.80-1.93) for any admissionhyponatremia compared with normonatremia. Adjusted HR for mild, moderate and severe hyponatremia compared with normonatremia was 1.67 (95\% Cl: $1.61-1.74), 2.27(95 \% \mathrm{Cl}: 2.14-2.41)$ and 2.35 $(95 \% \mathrm{Cl} 2.18-2.53)$, respectively.

\section{Conclusions}

- Admission-hyponatremia was associated with increased risk of death for all levels of hyponatremia, despite adjustment for older age and higher comorbidity level in hyponatremic patients.

- Risk of death increased with decreasing sodium levels.

- Even moderate hyponatremia was associated a more than 2-fold increased risk compared to normonatremia. 